\title{
A Study on the Influence of Nurses' Psychological Resilience on Work Stress in the Third-level Grade-A Hospital
}

\author{
Lin $\mathrm{Bo}^{1, \mathrm{a}}$, Ma Junbo ${ }^{1, \mathrm{~b}}$,Chi Keqiang ${ }^{2, \mathrm{c}^{*}}$ \\ ${ }^{l}$ Department of Economics and Management, Dalian University, Dalian, Liaoning, China \\ ${ }^{2}$ Dalian University, Dalian, Liaoning, China \\ alinbo@dlu.edu.cn \\ bmeasurable0828@163.com \\ c*chikeqiang@163.com
}

\begin{abstract}
Objective: To understand the psychological resilience and work pressure of nurses in the third-level grade-A hospital, and to explore the impact of psychological resilience of nurses in the third-level grade-A hospital on work pressure. To provide the basis for relieving the pressure of nurses in the third-level grade-A hospital, and to provide the improvement path for improving the psychological resilience of nurses. Methods: From March to April 2020, 457 nurses in the third-level grade-A hospital were investigated using the Work Stress Scale and the Psychological Resilience Scale. Results: The nurses who are unmarried, have worked 16-20 years or just started to work, and the younger ones have slightly higher mental resilience scores than other staff in the same group; The longer the daily working hours and the shorter the working years of nurses, the higher the work pressure score of other nurses in the same group; Psychological resilience and its dimensions are negatively correlated with work stress $(\mathrm{P}<0.001)$. Conclusion: The level of psychological resilience of nurses in the thirdlevel grade-A hospital is better, and the work pressure is in the middle or higher level; Different types of nurses have different levels of psychological resilience, among which marital status, working years, working hours per day and age may be the influencing factors; The better the psychological resilience level of the nurses in the third-level grade-A hospital is, the lower the work pressure level is.
\end{abstract}

Keywords: psychological resilience, work pressure, third-level grade-A hospital, nurse

\section{三甲医院护士心理弹性对工作压力的影响研究}

大连大学经济管理学院, 大连, 辽宁, 中国

大连大学, 大连, 辽宁, 中国

alinbo@dlu.edu.cn

${ }^{b}$ measurable0828@163.com

c*chikeqiang@163.com

\section{摘要}

目的: 了解三甲医院护士的心理弹性和工作压力现状, 探讨三甲医院护士心理弹性对工作压力的影响。 为缓解三甲医院护士压力提供依据, 为提高护士心理弹性提供改善路径。方法: 2020 年 3 月-4月, 利 用工作压力量表以及心理弹性量表，对三甲医院 457 名护士进行调查。结果：未婚、工作 16-20 年或 刚刚开始工作以及年龄较小的护士心理弹性得分略高于同组其他人员; 每天工作时间越长以及工作年 限越小的护士工作压力得分高于同组其他人员; 心理弹性及其维度与工作压力呈现负相关 $(\mathrm{P}<0.001)$ 。 结论: 三甲医院护士心理弹性水平较好, 工作压力处于中等略高水平; 不同类型护士心理弹性水平各 异, 其中婚姻状况、工作年限、每天工作时间、年龄可能为其影响因素; 三甲医院护士的心理弹性水 平越好，其工作压力水平越低。 
关键词: 心理弹性, 工作压力, 三甲医院, 护士

\section{1. 前言}

目前, 新型冠状病毒肺炎病毒在全世界范围内迅速 蔓延, 对全球各地人们的生命健康和财产都造成极大的 威胁和损失。各国各地的医务工作者作为抗击新型冠状 病毒肺炎病毒的中坚力量, 其重要性不言而喻。疫情的 暴发对这些医务工作者的医疗技术、护理技术和个人品 行都提出了进一步的要求。这对医务工作者的工作产生 强烈冲击, 因此有必要进一步探讨在内外部双重影响之 下的工作压力以及何种举措能缓解医务工作者的工作 压力。从国内的情况来看, 一方面, 改革开放以来, 随 着经济社会的发展, 我国男性和女性之间的工作结构发 生了一些变化, 大多数女性工作者从家庭走向职场, 从 “家庭妇女” 转变为 “职场女行”, 竞争越来越激烈, 职 业负担越来越繁重。另一方面, 受到传统价值观念和儒 家传统文化的影响, 大部分家务劳动还是落到女性身上。 随着医院逐步取消事业编制和二胎政策的全面放开, 我 国大约有几千万育龄妇女会受到这两项政策的影响。护 士本身具有高压、强专业的特点 ${ }^{[1]}$, 并且患者对护士的 护理技术、护理态度等要求十分严格。以女性为主要代 表的护士群体也正面临着来自工作、家庭、新型冠状病 毒肺炎病毒等内外部环境等各个方面的压力和挑战。若 长期处于这种工作压力之下，势必会对护士的护理技术、 护理态度产生影响 ${ }^{[2]}$ 。因此本研究将探讨三甲医院护士 的工作压力情况以及影响护士工作压力的影响因素。

工作要求-资源模型为我们回答上述问题提出了理 论基础。工作要求-资源模型认为, 当个体遇到来自外部 的各种工作要求时, 如: 工作压力, 那么个体此时就会 选择调动所拥有的工作资源, 如: 物质资源、精神资源、 社会资源等, 来弥补工作要求对个体的损耗和造成的不 良影响 ${ }^{[3]}$ 。心理弹性则代表了个体应对外部应激和刺激 事件的一种反应和调节能力。心理弹性是指个体在遭遇 外部应激和刺激事件后, 在多大程度上能够良好的适应 并迅速恢复的能力。心理弹性包括 3 个维度, 分别是: 坚㓞、乐观和力量。曾有学者在研究中表明心理弹性对 工作压力具有调节作用 ${ }^{[5-6]}$, 本研究将直接探讨二者之 间的影响关系。

综上所述, 本研究提出一个可能研究问题: 以三甲 医院护士为研究对象, 了解护士群体的心理弹性和工作 压力水平, 并探析护士的心理弹性与工作压力之间的关 系, 进而为医院管理与制定相关制度措施, 为缓解护士 工作压力提供科学的依据。

图 1 为本研究的理论模型。

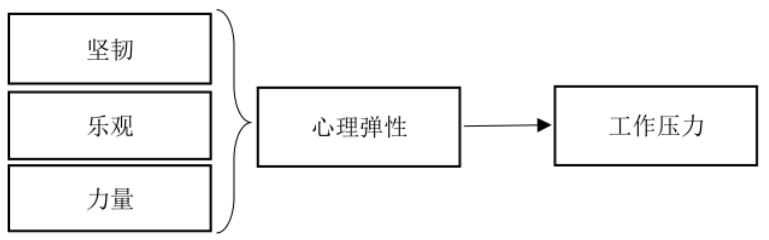

图 1 理论模型

\section{2. 资料来源与方法}

\section{1. 调查对象}

利用简单随机抽样方法, 选取三甲医院, 对愿意参 与此次调查的护士进行随机抽样调查。共发放问卷 488 份, 回收 488 份, 剔除信息失真等问卷后, 最终共 457 份有效问卷，有效回收率为 $93.6 \%$ 。

\section{2. 研究方法}

2020 年 3 月 -4 月，通过问卷星平台 (https://www. wjx. cn/), 以电子问卷形式对研究对象 进行调查。填写电子问卷前对研究对象详细说明填写要 求。为确保收集的数据真实有效, 设置同一 ID 只能填 写 1 份问卷。

\section{2. 1. 一般情况调查}

主要内容包括: 年龄、婚姻庄康、工作年限、工作 时长等个人基本信息。

\subsection{2. 工作压力量表}

采用由 House 和 Rizzo 等编制 ${ }^{[7]}$ 的工作压力量表。 该量表采用 Likert 5 级评分法计分, 得分越高说明护 士工作压力越大。本研究中该量表 Cronbach' $\mathrm{s}$ a 系 数为 0.818 , 具有良好的信度水平, 能够用于一般的理 论研究与分析。

\section{2. 3. 心理弹性量表}

心理弹性量表使用 Wang 等编制的心理弹性量表简 表 $^{[8]}$, 该量表共包含 10 个条目, 包括坚韧、乐观和力量 3 个维度。该量表采用 Likert 5 级评分法计分, 得分 越高说明心理弹性的水平越高, 本研究该量表的 Cronbach' $\mathrm{s}$ a 系数为 0.932 , 坚韧、乐观、力量 3 个 维度的 Cronbach' $\mathrm{s}$ a 系数分别为 $0.832 、 0.825$ 、和 0.911 , 具有较高的信度水平, 能够用于一般的理论研究 与分析。

\section{3. 统计学方法}

首先, 使用信度分析对所回收的问卷信度进行检验; 其次, 使用 $\mathrm{t}$ 检验、单因素方差分析等分析心理弹性和 工作压力在不同情况下的得分情况; 最后, 使用相关分 
析和回归分析探索心理弹性及其各维度对工作压力的 影响。均取 $\mathrm{P}<0.05$ 表示统计结果具有显著性差异。

\section{3. 结论}

\section{1. 不同条件下心理弹性和工作压力得分情 况}

不同婚姻状况和工作年限的护士心理弹性得分差 异均有统计学意义 $(P<0.05)$ 。经两两比较, 未婚护士 得分高于已婚护士; 工作 16-20 年以及刚刚开始工作的 护士得分高于其他分组的护士。尽管年龄组的得分差异 $P>0.05$, 但是相比较每天工作时间, 年龄组的显著性接 近 0.05 , 说明不同年龄的护士心理弹性得分差异也可能 具有统计学意义, 年龄越小的护士其心理弹性得分越高。 不同工作年限和每天工作时间的护士工作压力得分差 异均有统计学意义 $(P<0.05)$, 经两两比较, 工作年限 较短的护士得分高于较多的护士; 每天工作时间越久, 其工作压力水平越大, 详见表 1 。

表 1 不同条件下心理弹性和工作压力得分情况（总 分均值士标准差）

\begin{tabular}{|c|c|c|}
\hline 项目 & 心理弹性总分 & 工作压力总分 \\
\hline \multicolumn{3}{|l|}{ 婚姻状况 } \\
\hline 已婚 & $42.84 \pm 6.88$ & $24.31 \pm 5.03$ \\
\hline 未婚 & $43.61 \pm 5.94$ & $25.02 \pm 5.52$ \\
\hline $\mathrm{t}$ & 7. 921 & 2. 001 \\
\hline P & 0.005 & 0.158 \\
\hline \multicolumn{3}{|l|}{ 工作年限 } \\
\hline 5 年及以下 & $44.12 \pm 5.68$ & $24.86 \pm 5.89$ \\
\hline $6-10$ 年 & $42.99 \pm 6.77$ & $24.66 \pm 4.91$ \\
\hline $11-15$ 年 & $41.99 \pm 7.14$ & $24.91 \pm 4.62$ \\
\hline $16-20$ 年 & $44.90 \pm 6.16$ & $22.08 \pm 5.81$ \\
\hline 21 年及以上 & $41.74 \pm 6.81$ & $24.89 \pm 4.42$ \\
\hline $\mathrm{F}$ & 2.909 & 2.583 \\
\hline $\mathrm{P}$ & 0.021 & 0.037 \\
\hline \multicolumn{3}{|l|}{ 每天工作时间 } \\
\hline 5 小时及以下 & $46.00 \pm 0.00$ & $17.00 \pm 0.00$ \\
\hline 6-8 小时 & $43.08 \pm 6.81$ & $24.27 \pm 5.28$ \\
\hline 9-11 小时 & $43.39 \pm 6.08$ & $24.87 \pm 5.01$ \\
\hline 12 小时及以上 & $41.21 \pm 5.79$ & $28.50 \pm 4.35$ \\
\hline $\mathrm{F}$ & 0.544 & 3, 899 \\
\hline $\mathrm{P}$ & 0.653 & 0.009 \\
\hline \multicolumn{3}{|l|}{ 年龄 } \\
\hline 20 岁及以下 & - & - \\
\hline 21-30 岁 & $43.59 \pm 5.97$ & $24.97 \pm 5.58$ \\
\hline 31-40 岁 & $43.24 \pm 6.94$ & $23.95 \pm 5.08$ \\
\hline 41-50 岁 & $40.94 \pm 7.09$ & $25.34 \pm 4.49$ \\
\hline 51-60 岁 & $42.67 \pm 6.76$ & $24.11 \pm 3.51$ \\
\hline 61 岁及以上 & - & - \\
\hline $\mathrm{F}$ & 2.273 & 1. 677 \\
\hline $\mathrm{P}$ & 0.079 & 0.171 \\
\hline
\end{tabular}

\section{2. 相关因素分析}

首先, 从总体得分情况来看, 三甲医院护士心理弹
性与工作压力水平呈负相关 $(r=-0.38, P<0.01)$ 。

其次, 从人口统计学变量来看心理弹性与年龄呈现 负相关 $(r=-0.09, P<0.05)$; 压力知觉与每天工作时间 呈正相关 $(r=0.12, P<0.01)$ 。婚姻状况与工作年限 $(r=-$ $0.52, \mathrm{P}<0.01)$ 和年龄 $(r=-0.50, \mathrm{P}<0.01)$ 呈现负相 关; 年龄分别与工作年限之间呈正相关 $(r=0.86, P<$ $0.01)$, 与每天工作时间呈负相关 $(r=-0.10, P<0.05)$ 。 以上结果基本符合现实情况并为回归分析提供了基本 的数据支持。

\section{表 2 相关因素分析结果}

\begin{tabular}{ccccccc}
\hline 项目 & 1 & 2 & 3 & 4 & 5 & 6 \\
1 & 1 & & & & & \\
2 & $-0.52^{* * *}$ & 1 & & & & \\
3 & 0.05 & -0.08 & 1 & & & \\
4 & $-0.50^{* *}$ & $0.86^{* *}$ & $-0.10^{*}$ & 1 & & \\
5 & 0.06 & -0.09 & -0.01 & $-0.09^{*}$ & 1 & \\
6 & 0.07 & -0.05 & $0.12^{* *}$ & 0.03 & $-0.38^{* *}$ & 1 \\
\hline
\end{tabular}

注： 1 代表婚姻状况； 2 代表工作年限； 3 代表每天工作 时间； 4 代表年龄； 5 代表心理弹性； 6 代表工作压力。 *代表 $\mathrm{P}<0.05 ， * *$ 代表 $\mathrm{P}<0.01 ， * * *$ 代表 $\mathrm{P}<0.001$, 下同。

\section{3. 回归分析}

由表 3 可知，心理弹性与工作压力具有显著的负向 关系 $(\beta=-0.38, P<0.001)$ 。这说明护士的心理弹性水 平越高, 其工作压力感程度越低。

由表 4 可知, 心理弹性的 3 个维度均对工作压力具 有显著的影响, 其中, 坚韧维度负向影响工作压力 ( $\beta$ $=-0.34, P<0.001)$, 乐观维度负向影响压力知觉 $(\beta=-$ $0.34, \mathrm{P}<0.001)$, 力量维度负向影响压力知觉 $(\beta=-$ $0.36, \mathrm{P}<0.001)$ 。

\section{表 3 回归分析结果}

\begin{tabular}{cccc}
\hline \multirow{2}{*}{ 预测变量 } & \multicolumn{3}{c}{ 结果变量: 工作压力 } \\
\cline { 2 - 4 } & $\mathrm{B}$ & $\mathrm{t}$ & $95 \% \mathrm{CI}$ \\
\hline 心理弹性 & -0.38 & $-8.62^{* * *}$ & {$[-0.46,-0.29]$} \\
$\mathrm{R}$ & \multicolumn{3}{c}{0.38} \\
$\mathrm{R}^{2}$ & \multicolumn{3}{c}{0.14} \\
$\mathrm{~F}$ & \multicolumn{3}{c}{$74.244^{* * * *}$} \\
\hline
\end{tabular}

\section{表 4 各维度回归分析结果}

\begin{tabular}{|c|c|c|c|}
\hline \multirow{2}{*}{$\begin{array}{c}\text { 预测变 } \\
\text { 量 }\end{array}$} & \multicolumn{3}{|c|}{ 结果变量: 工作压力 } \\
\hline & $\beta$ & $\mathrm{t}$ & $95 \% \mathrm{CI}$ \\
\hline 坚㓞 & -0.34 & $-7.72^{* \star \star}$ & {$[-0.47,-0.28]$} \\
\hline 乐观 & -0.34 & $-7.75^{* * * *}$ & {$[-0.35,-0.21]$} \\
\hline 力量 & -0.36 & $-8.19^{* * * *}$ & {$[-0.35,-0.21]$} \\
\hline $\mathrm{R}$ & \multicolumn{3}{|c|}{ 坚㓞: 0.34 ; 乐观: 0.34 ; 力量: 0.36} \\
\hline $\mathrm{R}^{2}$ & \multicolumn{3}{|c|}{ 坚㓞: 0.12 ; 乐观: 0.12 ; 力量: 0.13} \\
\hline $\mathrm{F}$ & \multicolumn{3}{|c|}{ 坚㓞: $59.610^{* * *}$; 乐观: $60.078^{* * * * *}$; 力量: $67.007^{* * * *}$} \\
\hline
\end{tabular}




\section{4. 讨论}

本研究探讨了三甲医院护士心理弹性对工作压力 的影响, 结果表明: 未婚、工作 16-20 年或刚刚开始工 作以及年龄较小的护士心理弹性得分略高于同组其他 人员; 每天工作时间越长以及工作年限越小的护士工作 压力得分高于同组其他人员; 心理弹性与工作压力之间 呈现负相关。三甲医院护士心理弹性水平较好, 工作压 力处于中等略偏上的程度; 不同类型护士对待心理弹性 和工作压力态度各异, 其中婚姻状况、工作年限、每天 工作时间、年龄可能为其影响因素; 三甲医院护士的心 理弹性水平越好, 其工作压力水平越低。

\section{1. 三甲医院护士心理弹性水平良好, 工作压 力水平中等略高}

通过对 457 名护士进行调查分析, 发现其心理弹性 水平良好, 说明护士在应对压力与工作时有积极的心态 和能力。工作压力水平处于中等略高的水平。

在心理弹性方面，均分均介于有些符合与非常符合 之间, 这体现了三甲医院的护士群体心理弹性水平良好, 在遇到问题、面对压力时能够调动自身具备的各种资源, 以一种坚韧、乐观、自信的心态应对来自各方面的挑战, 并且及时他们收到了压力等应激事件或者外部刺激的 影响, 他们也能较快的从影响中恢复过来, 快速重新投 入工作当中。

在压力知觉方面, 护士是一种以女性为主题并且具 有高压性质的职业, 患者也十分重视护士的护理技能和 护理服务态度, 当这种来自病人与患者的期待和要求以 及自身对自己的要求越强烈, 护士在工作中所感受到的 以及自身带来的危机感与压力感就会越强烈。在本研究 中, 工作压力的得分情况处于中等略高的程度, 这说明 尽管在具备高水平的心理弹性的缓解下, 护士们能够以 一种积极的心态应对工作压力, 但是由于受到护士职业 特性的影响, 以及受到来自工作、家庭等各种外部条件 的影响, 使得护士长期处于一种应激的状态, 因此护士 仍能感受到很多来自工作中的压力。

\section{2. 心理弹性和工作压力的影响因素}

首先, 婚姻状况、工作年限和年龄可能为心理弹性 的影响因素。未婚和工作年限较短的护士其心理弹性水 平往往越高。原因可能为相比较已婚多年和工作时间较 长且稳定的护士相比, 这类群体刚刚开始事业的征程, 他们需要不断提升自己的能力来为今后的事业做好铺 垫和打下基础。面对压力和困难, 他们需要自己有能力 与独立面对和解决, 久而久之, 在不断的克服困难中他 们变得更加积极、乐观、自信和坚强, 因此这类群体的 心理弹性水平往往较高。

其次, 工作年限和工作时长可能为工作压力的影响 因素。工作年限较短和工作时长较多的护士其工作压力
越高。这类群体往往是医院中新入职或新晋护士的代表, 他们此刻正面临着来自工作和家庭的双重压力, 既面临 这结婚生子的考验, 又需要为了自己的工作不断拼搏努 力。因此, 这类护士的工作压力要高于其他类型的护士。

\section{3. 心理弹性与工作压力呈负相关}

在工作中感受到压力在所难免, 重要的是学会如何 自我调适, 自我克服, 自我发展。近几年来, 护士离职 的现象比比皆是, 护士抱怨工作的事件屡见不鲜, 尽管 我国正式注册的护士数量比较客观, 但是与发达国家相 比, 我国护士短缺的情况仍然十分严重, 这些都是护士 的工作压力过高所带来的不良后果。因此找出缓解护士 工作压力的方法以及如何提高护士自我应对压力的能 力显得尤为重要, 也应该作为各级医院制定相关规定政 策和考核护士的重要依据。本研究发现, 心理弹性以及 心理弹性的 3 个维度, 坚韧、乐观、力量, 均对工作压 力具有负向影响, 由此可见提高心理弹性水平能够降低 工作压力。相关研究表明, 个体所具备的资源, 包括: 物质资源、精神资源、社会资源、组织资源等，对于缓 解和削弱由于工作压力所引起的各种不良后果均有一 定的作用 ${ }^{[9]}$ 。心理弹性则代表了这样一种心理资源, 在 受到外部的刺激后, 能够通过自身所具备的各种资源弥 补刺激所带来的影响并迅速从影响中恢复, 重新投身于 工作中 ${ }^{[10]}$ 。在这个过程中, 个体会以一种积极乐观、坚 忍不拔和自信的方式应对压力和挑战。在医疗行业中, 心理弹性通常被看做是医务人员应对压力的重要因素, 因此如何提升心理弹性以缓解压力十分重要, 结合本研 究结果提示: 可以通过弹性排班等方式; 可以安排心理 素质的培训, 重点关注新入职的护士以及新晋护士的心 理状况; 也可以将心理弹性水平纳入到护士考核体系中 作为重要参考指标。总之, 医院在制定相关政策时可以 将心理弹性作为依据, , 通过提高护士心理弹性水平来 降低工作压力。

\section{REFERENCES}

[1] Orrung W A, Jakobsson U, Edberg A K. (2015) Job strain and stress of conscience among nurse assistants working in residential care. J Nurse Manag, 23(3):368-379.

[2] Nantsupawat A, Nantsupawat R, Kunaviktikul W, et al. (2016) Nurse burnout, nurse-reported quality of care, and patient outcomes in Thai hospitals. J Nurs Scholarsh, 48(1):83-90.

[3]Demerouti,E.,Bakker,A.B.,Nachreiner,F.,\&Schaufeli,W. B.(2001).The job demands-resources model of burnout.Journal of Applied Psychology,86(3),499-512.

[4] Campbell-Sills L, Cohan SL, Stein MB.(2006) Relationship of resilience to personality, coping, and psychiatric symptoms in young adults. Behav ResTher, 44 
(4) : $585-599$.

[5] Melnyk B M , Hrabe D P, Szalacha L A. (2013)Relationships among work stress, job satisfaction, mental health, and healthy lifestyle behaviors in new graduate nurses attending the nurse athlete program : a call to action for nursing leaders. Nurs Adm Q,37(4):278 - 285.

[6]Drury V, Craigie M, Francis K, et al. (2014)Compassion satisfaction, compassion fatigue, anxiety, depression and stress in registered nurses in Australia : phase 2 results. $\mathrm{J}$ Nurs Manag, 22 (4): $519-531$.

[7] House S, Rizzo R D. (1972) The Mechanisms of Job Stress and Strain. London, UK: Wiley.

[8] Wang L, Shi Z, Zhang Y, et al. (2010) Psychometric properties of the 10-item Connor-Davidson Resilience Scale in Chinese earthquake victims. Psychiat Clin Neuros, 64 (5): 499-504.

[9] Luthans,F. (2002)The need for and meaning of positive organizational behavior. Journal of Organizational Behavior, 23(6): $695-706$.

[10] Xanthopoulou, D., Bakker, A. B., Demerouti, E., et al. (2007)The role of personal resources in the job demands - resources model. International Journal of Stress Management, 14(2):121-141.

[11] Matos PS, Neushotz LA, Griffin MJ, et al. (2010)An exploratory study of resilience and job satisfaction among psychiatric nurses working in inpatient units $[\mathrm{J}]$. Int $\mathrm{J}$ Ment Health Nurs, 19(5):307-312. 